\title{
Atualização Eficiente da Decomposição LU na Programação de Lotes e Cortes ${ }^{1}$
}

G.M. BRESSAN², A.R.L. OLIVEIRA, Universidade de São Paulo, Instituto de Ciências Matemáticas e de Computação, Av. do Trabalhador São-carlense, 400, Cx.P. 668, 13560-970 São Carlos, SP, Brasil

Resumo. Neste trabalho consideramos o Problema Combinado, que está baseado na decisão de antecipar ou não a produção de certos produtos finais. Assim, este problema acopla dois problemas conhecidos de otimização: o dimensionamento de lotes e corte de estoque. O modelo matemático do problema combinado nos fornece uma matriz de restrições que, reordenada, possui formato bloco triangular, de modo que cause pequeno enchimento da matriz da base ao realizarmos uma decomposição $L U$, mas as implementações geralmente não exploram a estrutura matricial do problema. Apresentamos uma implementação em MATLAB que simula trocas de colunas da base e verifica a esparsidade da decomposição. A proposta de construção da base esparsa leva a bons resultados computacionais em comparação com implementações que não consideram a estrutura esparsa específica da matriz de restrições.

\section{Introdução}

As indústrias de manufatura têm sido estimuladas a tornar seus processos mais eficientes devido a aspectos econômicos e avanços computacionais. Isto incentiva o crescimento de modelos de otimização para o controle e planejamento de sistemas produtivos, motivando pesquisas acadêmicas.

O gerenciamento da produção dentro de uma indústria é responsável pelo planejamento e controle da transformação de matérias-primas em produtos finais. O sistema responsável por este gerenciamento denomina-se Planejamento e Controle da Produção, que coordena as atividades, desde a aquisição de matérias-primas até a entrega dos produtos finais. Este problema é bem conhecido na literatura e tem motivado várias pesquisas acadêmicas $[6,7]$, mas as suas implementações geralmente não exploram a estrutura matricial específica do problema.

O objetivo deste trabalho consiste na resolução dos sistemas lineares oriundos do método simplex, explorando a estrutura matricial inerente ao problema de programação de lotes e cortes. Este é um modelo cuja matriz de restrições possui

\footnotetext{
${ }^{1}$ Apoio FAPESP e CNPq

2 glauciab@sel.eesc.usp.br

3 aurelio@ime.unicamp.br
} 
uma estrutura bloco angular que é acoplado por restrições adicionais. Os blocos matriciais têm alto grau de esparsidade e pouco acoplamento, indicando que esta abordagem deve produzir bons resultados em termos de eficiência computacional.

A solução eficiente de sistemas lineares de grande porte é de fundamental importância na resolução de problemas de otimização. A solução pode ser obtida através de métodos genéricos, via decomposição LU das matrizes que formam as bases no método simplex, ou através da exploração da estrutura da classe de problemas a ser resolvida [4].

\section{O Problema de Corte de Estoque}

Suponha que várias barras estejam disponíveis para serem cortadas na produção dos diversos itens. Temos então que escolher quais barras devem ser cortadas, ou seja, temos um problema de seleção de objetos a serem cortados. Os objetos em estoque de mesmo tipo são disponíveis em grande quantidade, e podem ser de apenas um único tipo ou de vários tipos, havendo ou não limitação de estoque. A solução deste problema terá muitas peças em estoque igualmente cortadas para a produção dos diferentes tipos de itens. É denominado padrão de corte a forma como um objeto (peça) é cortado para a produção de itens demandados.

O motivo por estarmos utilizando o método Simplex com geração de colunas neste trabalho, é pelo fato de existir um número elevado de colunas nos padrões de corte, o que proporciona uma matriz de restrições extremamente grande. Por isso, ao invés de escolhermos dentre todas as colunas disponíveis, resolvemos um subproblema que, para o corte de estoque, consiste na geração de um padrão de corte que fará parte da solução do problema original. Consideramos que apenas $m$ colunas da matriz de restrições serão necessárias para descrever uma solução ótima, isto é, uma solução básica, de modo que não necessitamos armazenar toda a matriz.

Consideremos o problema onde barras de comprimento $L$ devem ser cortadas em pedaços $l_{i}, i=1, \ldots, P$. As colunas da matriz de restrições $A$ são bem determinadas por: $X=\left\{a=\left(\alpha_{1}, \alpha_{2}, \ldots, \alpha_{P}\right) \mid l_{1} \alpha_{1}+l_{2} \alpha_{2}+\ldots+l_{P} \alpha_{P} \leq L_{i}, \alpha_{i} \geq 0\right.$ e inteiro $\}$ [1]

Algumas colunas de $A$ são facilmente construídas, considerando-se padrões homogêneos, ou seja, cada padrão de corte produz quantidades de um único tipo de peça. Os vetores associados a eles constituem $P$ vetores linearmente independentes de $X: a_{i}=\left(0, \ldots, a_{i i}, \ldots, 0\right), i=1, \ldots, P$, onde $a_{i i}=\left[L / l_{i}\right]$, e $[x]$ é o maior inteiro menor ou igual a $x$. Assim, estas colunas formam uma submatriz diagonal:

$$
D=\left[\begin{array}{cccc}
a_{11} & 0 & \cdots & 0 \\
0 & a_{22} & \cdots & 0 \\
\vdots & \vdots & \ddots & \vdots \\
0 & 0 & \cdots & a_{P P}
\end{array}\right]
$$

O sub-problema pode ser escrito por:

$$
\max \sum_{i=1}^{p} y_{i} \alpha_{i}
$$




$$
\begin{gathered}
\text { s.a: } \sum_{i=1}^{p} l_{i} \alpha_{i} \leq L, \\
\alpha_{i} \geq 0 \text { e inteiro, } i=1, \ldots, P .
\end{gathered}
$$

Este é o modelo de um problema conhecido na literatura como Problema da Mochila, cuja motivação decorre da situação hipotética onde um muambeiro deseja carregar sua mochila com itens, cujos valores de compra são $l_{i}$. O valor total da compra não pode ultrapassar $L$. O muambeiro deseja maximizar seu lucro total. A solução do problema da mochila fornece uma coluna $\left(\alpha_{1}, \ldots, \alpha_{P}\right)$ tal que o custo relativo é mínimo. Portanto, resolvendo o problema de Corte de Estoque Unidimensional, a cada iteração do método simplex será necessário resolver o problema da mochila para determinar a coluna com o menor custo relativo. Mais detalhes sobre este problema podem ser vistos em [5, 9].

\section{O Problema Combinado}

O processo de programar a produção pode ser dividido em três etapas: A primeira delas define uma carteira de pedidos para um horizonte de planejamento finito (como dias, meses, etc.), especificando as quantidades dos produtos finais demandados e suas respectivas datas de entrega. A segunda etapa converte a demanda de produtos finais em demanda de peças. Deste modo, um tipo de peça pode ser utilizado por vários produtos finais diferentes, e as quantidades das peças necessárias são produzidas. Finalmente, a terceira etapa decide a quantidade de produtos finais que devem ser produzidos em cada período do horizonte de planejamento, minimizando os custos e as perdas ocorridas no processo de corte das placas em peças.

Frequentemente existem perdas de material no corte de peças. Esta perda tende a ser relativamente menor conforme a demanda de peças aumenta, devido a um melhor rearranjo dos padrões de corte nas placas. Portanto surge uma pressão econômica para fabricar alguns produtos antecipadamente para minimizar as perdas. Por outro lado, os custos de estoque exercem pressão oposta no sentido de retardar a produção. Baseado nesta decisão de antecipar ou não a produção de certos produtos finais surge o Problema Combinado [3], que acopla dois importantes problemas de otimização, o dimensionamento de lotes e o corte de estoque.

\subsection{Problema de Dimensionamento de Lotes e Problema de Corte de Estoque}

Vejamos os problemas de corte de estoque e de dimensionamento de lotes descrevendo separadamente cada um deles.

Problema de Dimensionamento de Lotes (PDL): Este problema consiste em planejar a quantidade dos itens a ser produzida em vários estágios, em cada período ao longo de um horizonte de tempo finito, de modo a atender a demanda e otimizar uma função objetivo, como minimizar os custos de produção e de estocagem. Um 
PDL pode ser classificado como monoestágio, onde os itens são produzidos independentemente, e multiestágio, em que as produções dos itens são dependentes. Para resolvermos este problema, podemos decompô-lo em $M$ subproblemas com apenas um produto final, que podem ser resolvidos, por exemplo, por programação dinâmica [8].

Problema de Corte de Estoque (PCE): Este problema consiste na otimização do processo de corte de placas em peças menores nas quantidades e dimensões demandadas. Define-se padrão de corte como o arranjo das peças dentro de cada placa. Algumas regras são necessárias para defini-lo, como cortes do tipo guilhotinado (onde cada corte feito sobre uma placa retangular produz dois novos retângulos), limitação de peças (cortes restritos ou irrestritos), número de estágios (é dito ser 2-estágios quando apenas uma mudança no sentido dos cortes guilhotinados é permitida: horizontal/vertical ou vertical/horizontal). Além disso, o problema será bidimensional quando duas dimensões são relevantes para cortagem. Para resolver este problema, podemos aplicar o Método Simplex em conjunto com a técnica de geração de colunas [2].

\subsection{Resolução Prática do Problema Combinado}

Em situações reais, a maioria das indústrias aborda esses dois problemas de forma separada, devido à alta complexidade do Problema Combinado. Inicialmente, são determinadas para cada período do horizonte de planejamento, as quantidades de cada produto final (tamanho do lote) a serem produzidas. A partir desta informação, determina-se, para cada período, a quantidade de peças de cada tipo a serem cortadas e os melhores padrões de corte são gerados. Entretanto, tratá-los de forma separada pode elevar os custos globais, principalmente se uma parcela significativa do custo do produto final é formada pelo material a ser cortado. Para resolvermos os problemas de Corte de Estoque e de Dimensionamento de Lotes separadamente, podemos aplicar a Heurística da Decomposição, que consiste em:

1. Inicialmente, resolver o PDL, em que é dada uma carteira de pedidos e realizamos o planejamento da produção, decidindo a quantidade de cada tipo de produto final a ser produzido em cada período do horizonte de planejamento, minimizando os custos de produção, estoque e preparação.

2. Como segundo passo, para cada período do horizonte de planejamento, resolvemos um problema de corte de estoque com restrições de capacidade, e assim determinamos a quantidade de placas cortadas conforme um certo padrão.

3. Finalmente, a função objetivo do problema combinado é dada pela soma das funções objetivos dos problemas PDL e PCE.

Apesar das dificuldades do modelo combinado quanto à integralidade das variáveis que representam a quantidade de placas cortadas num certo padrão e a grande quantidade de padrões de corte que podem ser gerados, esta abordagem fornece um ganho significativo nos custos globais, incentivando estudos nesta linha. 


\subsection{Formulação Matemática do Problema Combinado}

O Problema Combinado consiste em decidir a quantidade de produtos finais a serem produzidos em cada período do horizonte de planejamento tal que minimize os custos da produção e estocagem (PDL) e a quantidade de placas a serem cortadas para compor produtos finais (PCE). Este problema pode ser formulado como um modelo inteiro-misto que analisa o compromisso entre antecipar a produção de certos lotes de produtos finais para minimizar os custos no processo de corte e preparação, e o aumento dos custos de estocagem [3]. Neste mesmo trabalho também foi proposta a resolução do problema combinado via método simplex.

Uma consideração importante nos modelos reais é a disponibilidade da serra em cada período. Temos de assegurar que o tempo gasto para cortar as placas não excede o tempo disponível.

\subsubsection{O Modelo}

Uma forma de resolver o Modelo Combinado desconsidera a ocorrência de custos de preparação e relaxa a integralidade das variáveis que representam a quantidade de placas cortadas, o que pressupõe grandes quantidades de demanda. O modelo pode ser aplicado na indústria de móveis, onde placas de madeira devem ser cortadas na produção de itens. Consideramos que haja apenas um tipo de placa em estoque, $L \times W$, suficiente para atender a demanda. Definimos então o modelo da seguinte forma:

$$
\begin{aligned}
& \min \sum_{i=1}^{M} \sum_{t=1}^{T}\left(c_{i t} x_{i t}+h_{i t} e_{i t}\right)+\sum_{j=1}^{N} \sum_{t=1}^{T} c p y_{j t}+\sum_{p=1}^{P} \sum_{t=1}^{T} h p_{p t} e p_{p t}, \\
& \text { s.a: } \quad x_{i t}+e_{i, t-1}-e_{i t}=d_{i t}, \\
& \sum_{j=1}^{N} a_{p j} y_{j t}+e p_{p, t-1}-e p_{p t}=\sum_{i=1}^{M} r_{p i} x_{i t} \quad \forall t=1 \ldots T, \\
& \sum_{j=1}^{N} v_{j} y_{j t} \leq u_{t}, \\
& x_{i t}, e_{i t}, y_{j t}, e p_{p t} \geq 0 .
\end{aligned}
$$

Índices:

$t=1, \ldots, T$ número de períodos,

$p=1, \ldots, P$ número de diferentes tipos de peças a serem cortadas,

$j=1, \ldots, N$ número de diferentes padrões de corte,

$i=1, \ldots, M$ número de diferentes produtos finais demandados.

\section{Parâmetros:}

$c_{i t}$ : custo de produção do produto final $i$ no período $t$,

$h_{i t}$ : custo de estocagem do produto final $i$ no período $t$, 
$h p_{p t}$ : custo de estocagem da peça tipo $p$ no período $t$,

$d_{i t}$ : demanda do produto final $i$ no período $t$,

$r_{p i}$ : número de peças tipo $p$ necessárias para formar um produto $i$,

$v_{j}$ : tempo gasto para cortar uma placa no padrão de corte $j$,

$a_{p j}$ : número de peças tipo $p$ no padrão $j$,

$u_{t}$ : tempo máximo de operação da serra,

$c p$ : custo da placa a ser cortada.

\section{Variáveis:}

$x_{i t}$ : quantidade do produto final $i$ produzido no período $t$,

$e_{i t}$ : quantidade do produto final $i$ em estoque no fim do período $t$,

$e p_{p t}$ : quantidade da peça tipo $p$ em estoque no fim do período $t$,

$y_{j t}$ : quantidade de placas cortadas usando o padrão $j$ no período $t$.

Este é um modelo linear, por não ser considerada a integralidade das variáveis, porém permanece a dificuldade referente à grande quantidade de padrões de corte que podem ser gerados. Por simplicidade de notação reescrevemos o modelo da seguinte forma:

$$
\begin{aligned}
& \quad \min \sum_{t=1}^{T}\left(c_{t} x_{t}+h_{t} e_{t}\right)+\sum_{t=1}^{T} c p y_{t}+\sum_{t=1}^{T} h p_{t} e p_{t}, \\
& \text { s.a: } \quad x_{t}+e_{t-1}-e_{t}=d_{t}, \\
& -R x_{t}+A y_{t}+e p_{t-1}-e p_{t}=0 \quad \forall t=1 \ldots T, \\
& v^{T} y_{t} \leq u_{t}, \\
& x_{t}, e_{t}, y_{t}, e p_{t} \geq 0 \quad \text { onde } e p_{0}, e_{0} \text { são conhecidos, }
\end{aligned}
$$

$c_{t}=\left(c_{1 t}, c_{2 t}, \ldots, c_{M t}\right)$ e os demais parâmetros e variáveis $h_{t}, e_{t}, h p_{t}, e p_{t}, d_{t}, x_{t}$ e $y_{t}$ são definidos de forma similar, $v^{T}=\left(\begin{array}{llll}v_{1} & v_{2} & \ldots & v_{N}\end{array}\right)$,

$$
R=\left[\begin{array}{cccc}
r_{11} & r_{12} & \cdots & r_{1 M} \\
r_{21} & r_{22} & \cdots & r_{2 M} \\
\vdots & \vdots & \ddots & \vdots \\
r_{P 1} & r_{P 2} & \cdots & r_{P M}
\end{array}\right]
$$

e $A$ é uma matriz $P \times N$ tal que cada coluna corresponde a um padrão de corte.

\subsubsection{A Matriz de Restrições para o Modelo Combinado}

Reordenamos a matriz de restrições de modo que adquira formato bloco diagonal, invertendo as posições das variáveis $e_{t}$ e $x_{t}$. Além disso, acrescentamos as variáveis 
de folga $f_{t}=u_{t}-v^{T} y_{t}$ para $t=1 \ldots T$. Assim, a matriz dos coeficientes para o modelo tem a seguinte forma:

\begin{tabular}{cccccccccccc|ccc|c}
$e_{1}$ & $\ldots$ & $e_{T}$ & $x_{1}$ & $\ldots$ & $x_{T}$ & $y_{1}$ & $\ldots$ & $y_{T}$ & $e p_{1}$ & $\ldots$ & $e p_{T}$ & $f_{1}$ & $\ldots$ & $f_{T}$ & $d_{t}$ \\
\hline$-\mathrm{I}$ & $\ldots$ & 0 & $\mathrm{I}$ & $\ldots$ & 0 & 0 & & 0 & 0 & & 0 & 0 & $\ldots$ & 0 & $d_{1}-I_{0}$ \\
\hline $\mathrm{I}$ & $\ldots$ & 0 & 0 & $\ldots$ & 0 & 0 & & 0 & 0 & & 0 & 0 & $\ldots$ & 0 & $d_{2}$ \\
\hline & & & & & & 0 & & 0 & 0 & & 0 & & $\ldots$ & & $\ldots$ \\
\hline 0 & $\ldots$ & 0 & 0 & $\ldots$ & 0 & 0 & & 0 & 0 & & 0 & 0 & $\ldots$ & 0 & $d_{t-1}$ \\
\hline 0 & $\ldots$ & 0 & 0 & $\ldots$ & 0 & 0 & & 0 & 0 & & 0 & 0 & $\ldots$ & 0 & $d_{t}$ \\
\hline & $\ldots$ & & $\ldots$ & & & & & & & & & & $\ldots$ & & $\ldots$ \\
\hline 0 & $\ldots$ & $-\mathrm{I}$ & 0 & $\ldots$ & $\mathrm{I}$ & 0 & & 0 & 0 & & 0 & 0 & $\ldots$ & 0 & $d_{T}$ \\
\hline 0 & $\ldots$ & 0 & $-\mathrm{R}$ & $\ldots$ & 0 & $\mathrm{~A}$ & $\ldots$ & 0 & $-\mathrm{I}$ & $\ldots$ & 0 & 0 & $\ldots$ & 0 & 0 \\
\hline 0 & $\ldots$ & 0 & 0 & $\ldots$ & 0 & 0 & $\ldots$ & 0 & $\mathrm{I}$ & $\ldots$ & 0 & 0 & $\ldots$ & 0 & 0 \\
\hline 0 & $\ldots$ & 0 & & $\ldots$ & & & $\ldots$ & & & $\ldots$ & & & $\ldots$ & & 0 \\
\hline 0 & $\ldots$ & 0 & 0 & $\ldots$ & $-\mathrm{R}$ & 0 & $\ldots$ & $\mathrm{A}$ & 0 & $\ldots$ & $-\mathrm{I}$ & 0 & $\ldots$ & 0 & 0 \\
\hline 0 & $\ldots$ & 0 & 0 & $\ldots$ & 0 & $v^{T}$ & $\ldots$ & 0 & 0 & $\ldots$ & 0 & -1 & $\ldots$ & 0 & $u_{1}$ \\
\hline 0 & $\ldots$ & 0 & 0 & $\ldots$ & 0 & 0 & $\ldots$ & 0 & 0 & $\ldots$ & 0 & 0 & $\ldots$ & 0 & $u_{2}$ \\
\hline & $\ldots$ & & & $\ldots$ & & & $\ldots$ & & & $\ldots$ & & $\ldots$ & $\ldots$ & & $\ldots$ \\
\hline 0 & $\ldots$ & 0 & 0 & $\ldots$ & 0 & 0 & $\ldots$ & $v^{T}$ & 0 & $\ldots$ & 0 & 0 & $\ldots$ & -1 & $u_{T}$ \\
\hline
\end{tabular}

Tabela 1: Matriz de Restrições para o Problema Combinado

É vantajoso que as colunas básicas respeitem este ordenamento, pois a matriz de restrições possuirá formato bloco diagonal, de modo que causará o menor preenchimento da base ao realizarmos sua decomposição LU.

\section{Base inicial para a Matriz de Restrições}

Uma base inicial pode ser tomada considerando vazios os estoques de peças e de produtos finais e considerando a matriz dos padrões de corte como diagonal, através da escolha dos padrões homogêneos. Desta forma, obtemos a seguinte base inicial:

\begin{tabular}{|cccc|cccc|c|}
$x_{1}$ & $x_{2}$ & $\ldots$ & $x_{T}$ & $y_{1}$ & $y_{2}$ & $\ldots$ & $y_{T}$ & $\mathrm{f}$ \\
\hline$I_{M \times M}$ & 0 & $\ldots$ & 0 & 0 & 0 & $\ldots$ & 0 & 0 \\
0 & $I_{M \times M}$ & $\ldots$ & 0 & 0 & 0 & $\ldots$ & 0 & 0 \\
& & $\ldots$ & & & & $\ldots$ & & \\
0 & 0 & $\ldots$ & $I_{M \times M}$ & 0 & 0 & $\ldots$ & 0 & 0 \\
\hline$-R_{P \times M}$ & 0 & $\ldots$ & 0 & $D_{P \times P}$ & 0 & $\ldots$ & 0 & 0 \\
0 & $-R_{P \times M}$ & $\ldots$ & 0 & 0 & $D_{P \times P}$ & $\ldots$ & 0 & 0 \\
0 & 0 & $\ldots$ & & & & $\ldots$ & & \\
0 & 0 & $\ldots$ & 0 & $v_{B_{1 \times P}}^{T}$ & 0 & $\ldots$ & 0 & \\
0 & 0 & $\ldots$ & 0 & 0 & $v_{B_{1 \times P}}^{T}$ & $\ldots$ & 0 & $-I$ \\
0 & 0 & $\ldots$ & 0 & 0 & 0 & $\ldots$ & $v_{B_{1 \times P}}^{T}$ &
\end{tabular}

sendo $D$ uma submatriz diagonal de $A$ de dimensão $P \times P$ e $v_{B_{1 \times P}}$ representa o vetor cujas colunas são correspondentes às colunas de $D$. 
As colunas são todas linearmente independentes, ou seja, a matriz base inicial é não singular, pois esta matriz é triangular inferior e todos os elementos da diagonal são diferentes de zero. Não é necessário decompor esta base.

\subsection{Identificação do Tipo das Colunas}

Dadas a coluna que sai da base e a que entra, podemos identificar a qual bloco pertencem estas colunas, utilizando a posição do primeiro e do último elemento não nulo, pelo algoritmo descrito abaixo. Portanto, são necessárias poucas alterações em uma implementação pré-existente que não considera a estrutura particular da matriz de restrições. Nosso objetivo é incluir a decomposição LU nesta implementação, bastando que as colunas que entram e saem da base sejam fornecidas.

Dada uma coluna $c$, obtemos as posições de seu primeiro e último elementos não nulos $k_{1}$ e $k_{2}$ :

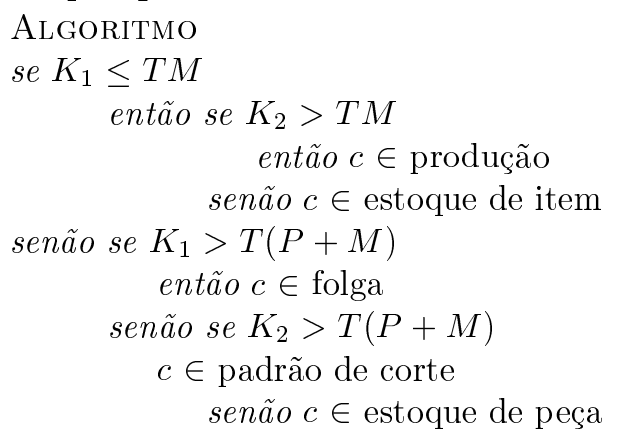

\section{Experimentos Numéricos}

O objetivo destes experimentos é verificar a viabilidade das idéias apresentadas neste trabalho. No experimento numérico descrito a seguir consideramos: 6 períodos de tempo, 2 produtos finais, 5 peças diferentes e 60 padrões de corte.

Com esses parâmetros, a matriz de restrições possui dimensão $48 \times 420$, sendo 360 colunas correspondentes à padrões de corte. Para desenvolvermos o experimento, definimos as matrizes $B_{1}, B_{2}$ e $B_{3}$. Inicialmente todas elas correspondem à base inicial. Vamos simular iterações do método simplex escolhendo aleatoriamente uma coluna para sair da base e uma para entrar na base. A cada iteração as três matrizes contêm as mesmas colunas básicas, embora em ordem diferente. As colunas em $B_{1}$ respeitam o ordenamento descrito na Tabela 1 . Na matriz $B_{2}$ sempre colocamos a coluna que entra na base na posição da coluna que sai e na matriz $B_{3}$ a coluna que entra na base ocupa a última posição da matriz.

\subsection{Critério para Trocas de Colunas}

Realizamos trocas das colunas básicas por colunas não básicas, testando a singularidade e a esparsidade. Sorteamos uma coluna que sairá da base e uma coluna que 
será inserida. Se a nova base for não singular, atualizamos as posições das colunas básicas em $B_{1}, B_{2}$ e $B_{3}$, da forma descrita anteriormente; caso contrário, sorteamos outra coluna para entrar na base até que encontremos uma matriz não singular. Efetuamos a seguir a decomposição LU de $B_{1}, B_{2}$ e $B_{3}$, e calculamos o número de elementos não nulos dessas decomposições.

Esperamos que $B_{1}$ seja mais esparsa que $B_{2}$ e $B_{3}$, porque ela é ordenada de forma que sua decomposição cause menor preenchimento na matriz.

\subsection{Resultados Observados}

Apresentamos nas tabelas seguintes os resultados obtidos pelos experimentos numéricos. Os valores min, max e média nas tabelas 2 e 3 representam, respectivamente, o mínimo, o máximo e a média do número de elementos não nulos das matrizes $L$ e $U(n n z(L+U))$ da decomposição das bases correspondentes. A singularidade da matriz é verificada pela função rcond() do MATLAB, e o cálculo da decomposição $L U$ foi realizado usando o comando interno $l u()$, que reordena as linhas para a seleção do pivô. Antes da primeira troca de colunas, as matrizes $B_{1}, B_{2}$ e $B_{3}$ são iguais. Os resultados apresentados consideram diversos números de iterações.

Os resultados obtidos para as 500 primeiras iterações foram:

\begin{tabular}{|c|c|c|c|}
\hline $\mathrm{nnz}(\mathrm{L}+\mathrm{U})$ & $B_{1}$ & $B_{2}$ & $B_{3}$ \\
\hline $\min$ & 280 & 279 & 286 \\
\hline $\max$ & 408 & 478 & 388 \\
\hline média & 359.3 & 396.1 & 355.3 \\
\hline
\end{tabular}

Tabela 2: Número de elementos não nulos da decomposição para 500 iterações

Os resultados obtidos para as 10000 primeiras iterações foram:

\begin{tabular}{|c|c|c|c|}
\hline $\mathrm{nnz}(\mathrm{L}+\mathrm{U})$ & $B_{1}$ & $B_{2}$ & $B_{3}$ \\
\hline $\min$ & 275 & 278 & 281 \\
\hline $\max$ & 401 & 573 & 411 \\
\hline média & 335.7 & 370.1 & 342.8 \\
\hline
\end{tabular}

Tabela 3: Número de elementos não nulos da decomposição para 10000 iterações

\section{Conclusões e Perspectivas Futuras}

Podemos concluir que a estratégia $B_{1}$ é melhor sob qualquer aspecto, pois, por exemplo, para 10000 iterações, uma das respostas obtidas foi que o máximo de elementos não nulos na decomposição de $B_{1}$ foi 401 , enquanto que para $B_{2}$ foi 573 e para $B_{3}$ foi 411 . Como a base possui 2304 elementos, esses valores correspondem, respectivamente, a $83 \%, 75 \%$ e $81 \%$ de esparsidade. Podemos observar que a estratégia $B_{3}$ assume uma posição intermediária entre $B_{1}$ e $B_{2}$. Desta forma, $B_{1}$ 
contribui para a redução de tempo computacional, podendo resolver problemas de otimização linear de maneira mais eficiente.

Nosso próximo objetivo é implementar rotinas da decomposição LU para a base da matriz de restrições, considerando a estrutura esparsa da matriz e integrar esta implementação com códigos já existentes, mas que não exploram a esparsidade da matriz de restrições.

\begin{abstract}
In this work the combined problem which connects the lot sizing and the cutting stock problems is considered. We study the properties of the matrix of constrains and how to factorize the bases without losing sparsity in the simplex method context. Numerical results of an MATLAB implementation simulate simplex iterations and verify the sparsity pattern of the factorizations. We conclude that the proposal of construction of sparse bases leads to good computational results in comparison with implementations which do not consider the sparse structure of the matrix of constrains.
\end{abstract}

\title{
Referências
}

[1] M. Arenales e R. Morabito, "Modelos e Métodos Básicos dos Problemas de Corte - O Problema de Corte e Empacotamento e Aplicações Industriais", Texto de Mini-curso do XX CNMAC, Gramado-RS, 1997.

[2] P. Gilmore e R. Gomory, Multistage cutting stock problems of two and more dimensions, Operation Research, 14 (1965), 1045-1074.

[3] M.C.N. Gramani, "Otimização do Processo de Cortagem acoplado ao planejamento da produção", Tese de Doutorado, Densis-Unicamp, 2001.

[4] D.G. Luenberger, "Linear and Nonlinear Programming", Addison-Wesley, 1984 .

[5] F.P. Marques, "O Problema da Mochila Compartimentada", Dissertação de Mestrado, ICMC-USP, 2000.

[6] S.L. Nonas e A. Thorstenson, A combined cutting-stock and lot-sizing problem, Operation Research, 120, No 2 (2000), 327-342.

[7] M. Reinders, Cutting stock optimization and integral productions planning for cintralized wood processing, Mathematical Computer Modeling, 16, No.1 (1992), 1992.

[8] H.M. Wagner e T.M. Whitin, Dynamic version of the Economic Lot size Model, Management Science, 5, No. 1, (1958), 89-96.

[9] H. Yanasse e N. Soma, O problema de Corte e Empacotamento e Aplicações Industriais: O problema da mochila, em XX Congresso Nacional de Matemática Aplicada e Computacional, Gramado-RS, 1997. 